\title{
Traffic safety management based on integrated assessment of safety level
}

\author{
Philipp Sukhov ${ }^{1, *}$, Vladimir Popov ${ }^{1}$, and Viktor Filippov ${ }^{1}$ \\ ${ }^{1}$ Russian University of Transport (MIIT), 9b9 Obrazcova Street, Moscow, 127994, Russia
}

\begin{abstract}
The volumes of freight and passenger traffic on the railways of the Russian Federation are increasing every year. Along with the increase in the transportation work, the likelihood of occurrence of undesirable incidents in railway transport increases. The assessment of the level of comprehensive (integrated) safety is an important scientific and technical problem in the current conditions of the functioning of railway transport. The methods used to assess the level of safety do not always meet the requirements of complexity in view of comparison of various-scale violations of traffic safety (crash, accident, faulty operation). The paper proposes a mathematical method for assessing the level of integrated safety in railway transport and calculation of the safety index of train traffic. For the calculation, statistical data for a certain period are used. These data summarized in a specialized dictionary of traffic safety violations, the mathematical treatment of which provides information on the level of integrated safety in railway transport in the Russian Federation. The proposed index allows determining the effectiveness of management decisions in the field of train traffic safety, as well as assessing the effectiveness of implementing certain innovations in the field of traffic safety. It is possible to use data on integrated traffic safety to reduce insurance premiums for cargo insurance on railways.
\end{abstract}

\section{Introduction}

Integrated assessment of technical safety is an urgent problem for all types of land, water, and air transport [1]. The need for safety is approved at the legislative level of the Russian Federation [2,3]. According to the official data of JSC "Russian Railways", the annual growth in the volume of transportation by railways of the Russian Federation is from 5 to 10 percent. Because of the constant increase in transportation, the load on the rolling stock and railway transport infrastructure is increasing. Due to the increasing load, the issues of ensuring the safety of transportation process and, at the same time, assessing the level of safety of transport in general are becoming more urgent. The railway transport system is a complex integrated [4] sociotechnical system.

In papers [5-7], it is noted that in order to implement the strategy of sustainable development and ensure the safety of transport, along with the concept of strategic risks, it is

\footnotetext{
* Corresponding author: Philipp.sukhov@mail.ru
} 
necessary to analyze and manage on the basis of a comprehensive and integrated index of the safety level.

To date, the total number of traffic safety violations (TSV) - the sum of all the accidents listed in $[8,9]$ that occurred during the reporting year, is usually used in the Russian railway transport system for assessing the level of technical safety. According our assessment, [10] the difference in scales of such TSVs as an accident, crash and other less severe consequences of TSV (crashes and accidents are rare types of TSV, and faulty operation - mass types of TSV) does not allow applying additive procedures as a quantitative assessment of the traffic safety level.

The reason for the different scales of different types of TSV lies in the nature of their occurrence. The nature of the occurrence of crashes and accidents is mainly bifurcational in character: at the point of possible branching of the process of train movement, a huge number of random factors that cannot be controlled determines by which scenario the process will continue. This is the practical impossibility of predicting these events and the difficulty of identifying the causes of their occurrence.

To obtain the indices of the integrated assessment of the safety level of train traffic, we developed the following mathematical model.

\section{Materials and methods}

The list of TSVs for a year is a material document (text), which is an information system with weak connections that reflects the state of the real system of "traffic safety on the railways" in a modified and transformed form using a special dictionary (Figure 1). This dictionary consists of different types of TSV [11] on the railways, which include the concepts of crash, accident, special faulty operation and faulty operation, the causes of crashes, accidents, derailments and collisions of trains differentiated by the types of activity of railway transport facilities. For stable systems with weak connections of any nature (bio-techno- and informocenosis) that are capable of development, isomorphism of structure and presence of the emergence (integrity) property are characteristic. These properties are manifested in their stable species diversity in accordance with Zipf's law [12].

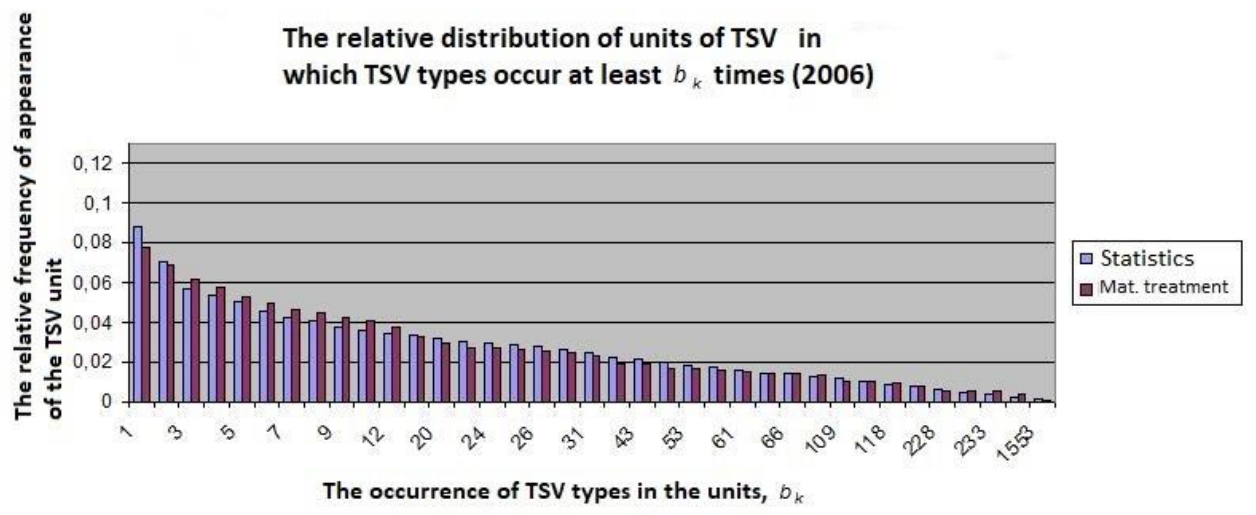

Fig. 1. Relative distribution of units of TSV in which TSV types occur at least $b_{k}$ times (2006).

We will consider [10] the set $\mathrm{U}$ of all traffic safety violations (TSV) for the year as a system with weak connections. Each TSV $u$ is, on the one hand, an individuality (an individual) - an element of the set $\mathrm{u} \in \mathrm{U}$, and on the other hand, a representative of the type $\mathrm{u} \in \mathrm{S}$, where $\mathrm{S}$ is the set of TSV types. Then the common list of TSV $\mathrm{u}$ - the text of the volume 
$T=|U|$, the common list of types of TSV $\mathrm{s} \in \mathrm{S}$ - the dictionary with the volume $V=|S|$. Based on the results of [13], and in accordance with the provisions of [8,9], the following dictionary of TSV was formed:

- Transport accident (TA) - various types of derailment or collision of trains and rolling stock, harming life and health of people, property and the environment. TA - represented by a triune structure - "Reason - type of derailment or collision - consequences".

- Dangerous transport event (DTE) - an event in the train or shunting work that immediately precedes the TA - "TA cause" or creating a threat of TA's occurrence - "precondition of TA", as a result of which, due to randomness or actions of the personnel, TA didn't occur.

- Faulty operation (FO) - the result of special measures to counter the threat to traffic safety with the aim of its minimizing or malfunctioned technical means that do not directly threaten traffic safety, which indirectly influence the occurrence of DTE.

Facilities of JSC "RZD" - responsible for the occurrence of a traffic safety violation.

Let's assume that $n_{i}=n_{i}(j), n_{i} \subset S, i=1,2,3, \ldots$ is the number of TSV types having exactly $j$ representatives $\left\{u_{1}, u_{2}, u_{3}, \ldots, u_{j}\right\} \subset U$. We form classes $a_{i}=j, \quad j=1,2,3, \ldots$, each of which contains $n_{i}=n_{i}\left(a_{i}\right)$ types of TSV represented by an equal number of individuals. From TSV classes of repeatability $a_{i}$, in each of which there are $n_{i}=n_{i}\left(a_{i}\right)$ types, we form TSV units $b_{k}=a_{i}, k=i, \mathrm{i}=1,2,3, \ldots, \mathrm{N}$. Assume that in each unit $b_{k}$ there are $(N+1-k)$ classes and, respectively, $m_{k}\left(b_{k}\right)=\sum_{i=k}^{N} n_{i}\left(a_{i}\right)$ types of TSV, i.e. in each unit $b_{k}$, such types of TSV which occur at least $b_{k}$ times are presented.

The volume of representatives of types in the units of TSV:

$$
W=|m|=\sum_{k=1}^{N} m_{k}=\sum_{k=1}^{N} \sum_{i=k}^{N} n_{i}\left(a_{i}\right)
$$

then the relative frequency of the appearance of TSV unit:

$$
\Omega_{k}\left(b_{k}\right)=m_{k}\left(b_{k}\right) / W
$$

under condition $\sum_{k=1}^{N} \Omega_{k}\left(b_{k}\right)=1$.

According to the actual data, statistical dependencies $\Omega_{k}=f\left(b_{k}\right)$ were constructed. These dependencies can be considered as messages that carry information about the state of the real system of "Traffic safety on the railways".

The mathematical treatment of these messages by the maximum likelihood method using the fitting criterion $\chi^{2}$ showed the existence of a stable pattern in the distribution of $\Omega_{k}\left(b_{k}\right)$ . This pattern has the following form:

$$
\Omega_{k}=\frac{\alpha}{b_{k}^{\beta}} \exp \left(-\gamma \ln ^{2} b_{k}\right)
$$

For statistical and theoretical dependencies of $\Omega_{k}=f\left(b_{k}\right)$, the entropy of these messages was calculated $H=-\sum_{k=1}^{N} \Omega_{k} \ln \Omega_{k}$ (Figure 2), which can be interpreted as 
a measure of the organization of the "Traffic safety on railways" system, and the indicator of information message evenness $I_{0}=H / \ln W$ (Figure 3), which is a quantitative assessment of the level of organized traffic safety on railways.

\section{Level of organization of traffic safety on the railways of Russia}

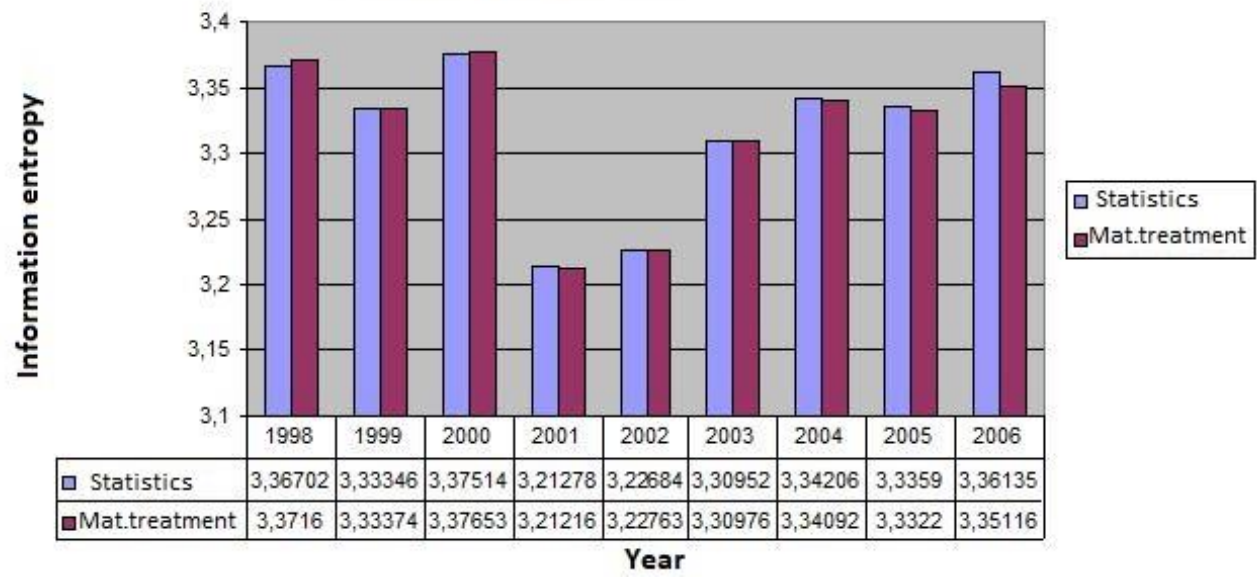

Fig. 2. Information entropy (measure of organization) of traffic safety on the railways of Russia.

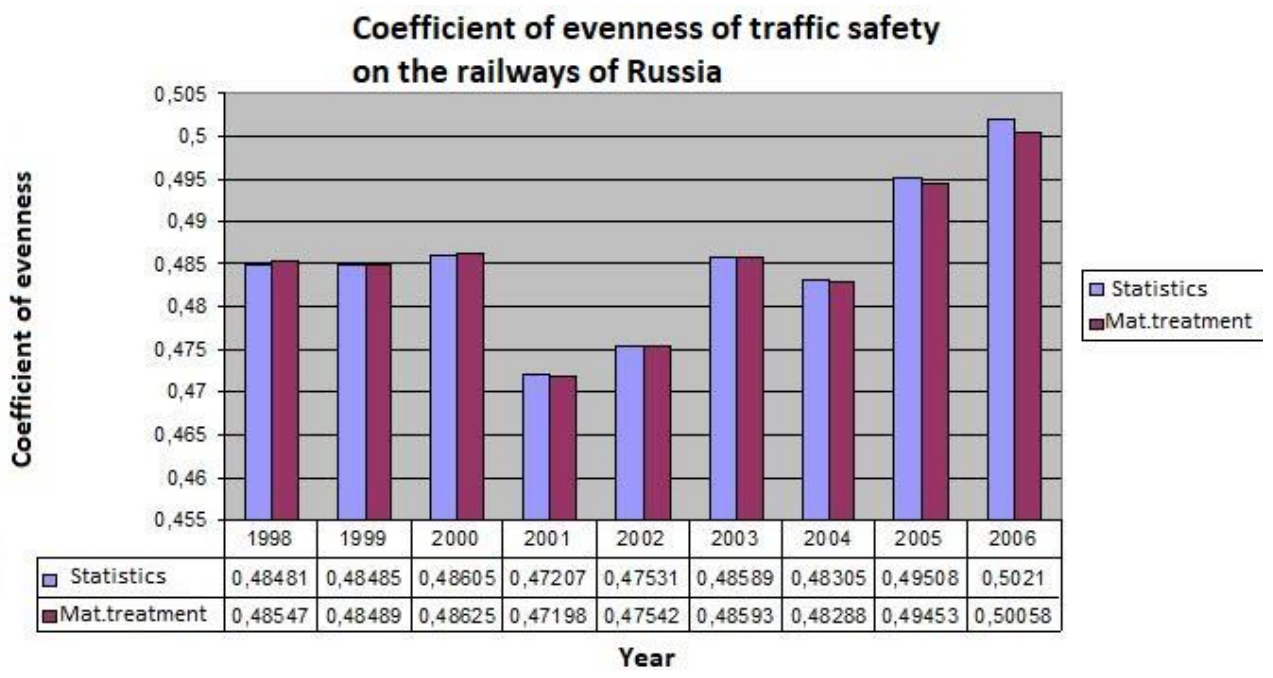

Fig. 3. Coefficient of evenness of traffic safety on the railways of Russia.

Computational experiments showed that a decrease in the number of rare types of TSV (crashes, accidents, derailments and collisions of trains, etc.) or an increase in the number of mass types of TSV (locomotive failure, which caused the train to delay by more than 1 hour, the uncoupling of the freight car because of malfunction of roller boxes, etc.), while maintaining the number of classes and units of TSV, leads to an increase in the value of $\mathrm{N}$ information content of the message. Conversely, an increase in the number of rare types of TSV or a decrease in the number of mass types of TSV, while maintaining the number of 
classes and units of TSV, leads to a drop in the value of N. The increase in the number of types, classes and units of TSV also leads to an increase in the value of N, i.e. the information content of the message increases with increasing level of detail in the detection of TSV.

Integrated quantitative assessment of the level of traffic safety on the railways (traffic safety index) depends on the combination of administrative and organizational measures and technical means for managing the train traffic, capital costs for new equipment, and operating costs for the repair and maintenance of rolling stock and railway infrastructure.

Based on the following heuristic assumptions, a general view of the traffic safety index for railway transport was obtained $U_{T S}$ :

- $U_{T S}$ depends on the reduced working capacity $C_{r e d}$ (billion tkm gross per year per 1

$\mathrm{km}$ of the operational length of the railway), since the reduced output of railway transport, with all other conditions being equal, is linearly related to its operational length, and the evenness factor $I_{0}$, and $U_{T S}=0$ with $C_{\text {red }}=0$ and $I_{0}=0$.

- The rate of change of $U_{T S}$ from the value of the working capacity $C_{r e d}$ for small values of the working capacity $C_{\text {red }}$ must be the largest, and with increasing value of $C_{r e d}$, should tend to zero because of the increasing complexity of the transport process, with all other conditions being equal. Or the derivative $\frac{d U_{T S}}{d\left(C_{\text {red }} / C_{\text {red } 0}\right)} \approx I_{0}$ with $C_{\text {red }}$ close to zero and $\frac{d U_{T S}}{d\left(C_{\text {red }} / C_{\text {red } 0}\right)} \rightarrow 0$ with sufficiently large $C_{r e d}$, in the limit $\lim _{\substack{C_{r e d} \rightarrow \infty \\ I_{0} \rightarrow 1}} U_{T S}=1$. Here, $C_{\text {redo } 0}$ is the base value of the reduced working capacity.

Integrated quantitative assessment of the level of traffic safety on the railways (the index of the level of traffic safety) has the following form:

$$
U_{T S}=-\frac{\sum_{k=1}^{k=N} \Omega_{k} \ln \Omega_{k}}{\ln W} \cdot\left[1-\exp \left(-C_{r e d} / C_{r e d 0}\right)\right]
$$

\section{Results}

To approbate the proposed mathematical model, the traffic safety index for 1998-2006 was calculated. This period was chosen because of the most complete statistics of TVS types. The values of $U_{T S}$ are calculated from the ratio (3) on the basis of statistical data (formula (1)) and their mathematical treatment (formula (2)). The results of the studies are shown in Fig.4.

Using the proposed mathematical model, it is possible to make a forecast of $U_{T S}$ based on statistical data for the previous periods and to perform their mathematical treatment using the maximum likelihood method. Computational experiments have shown that the fitting criterion $\chi^{2}$ makes it possible to apply a polynomial dependence of the third degree for extrapolation of $U_{T S}$ to subsequent years (the reliability of the approximation $\mathrm{R}^{2}$ for statistical data is about 0.974 , for data obtained by mathematical treatment is about 0.978 ). 


\section{Level of traffic safety on the railways of Russia}

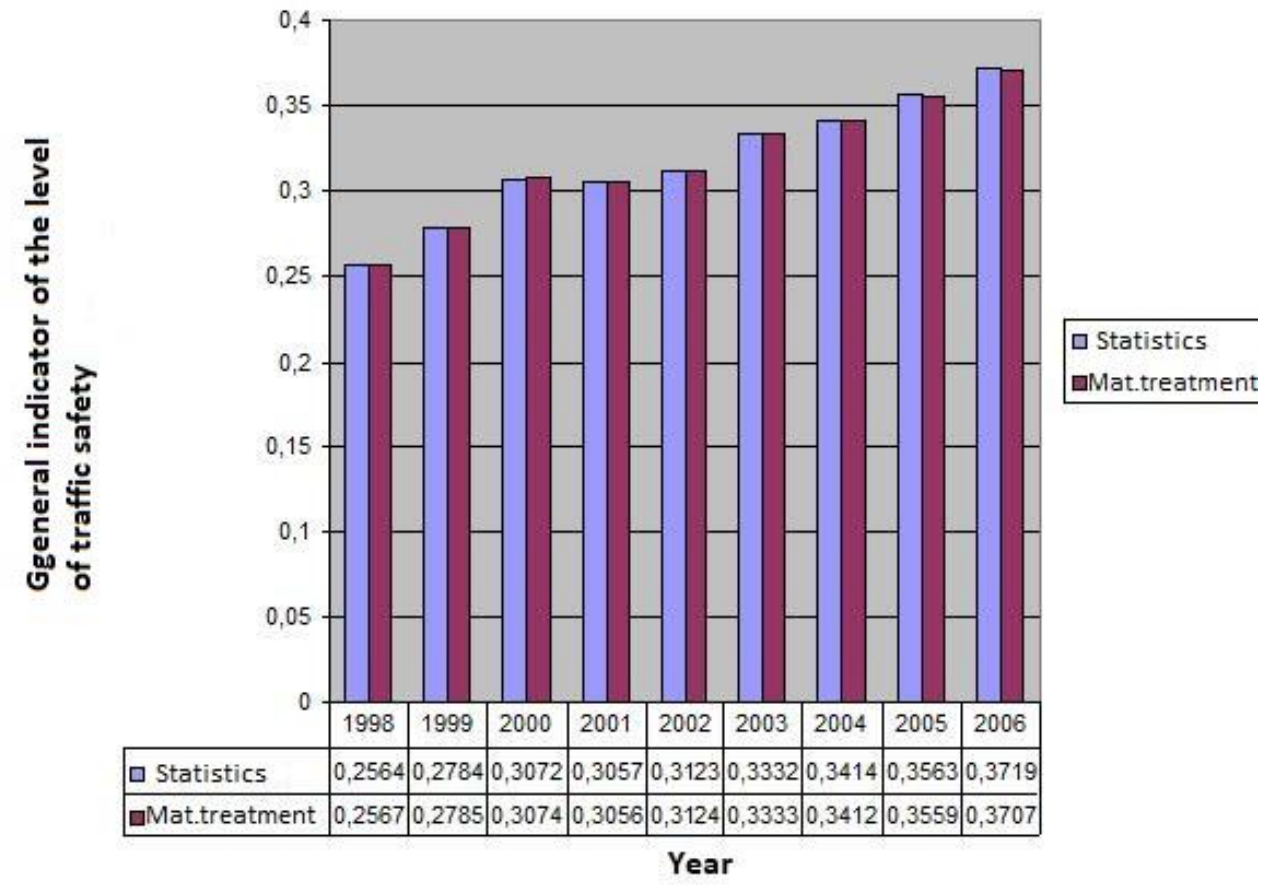

Fig. 4. Level of traffic safety on the railways of Russia.

The obtained results show that the growth rate slows down after 2000, and in 2001 it decreases compared to 2000, despite the annual constant decrease in the total number of TSV. This conclusion is confirmed by the results of paper [14], which shows a drop in the rate of growth in operating costs since 2000 .

Moreover, it is noted that the number of depots has decreased, the hauls of locomotive circulation and the service areas of the maintenance depots have extended, which to some extent affected the increase in the number of derailment of freight and passenger trains in 2001 , i.e. led to a decrease in the level of traffic safety on the railways in Russia.

In the future, the implementation of activities of the annual program of JSC "RZD" to improve traffic safety allowed first stabilizing and then ensuring a constant increase in the level of train traffic safety. This trend is confirmed by the performed forecast.

\section{Conclusions}

Based on the papers $[10,12,15]$, the authors developed a traffic safety level index $U_{T S}$ that allows determining the response of the real system of "Traffic safety on the railways" after the adoption and implementation of the management decision.

Carrying out computational experiments, it is possible to create a forecast of the traffic safety index for various management decisions, which should allow choosing the most effective management solution in the field of traffic safety. 


\section{References}

1. L.N. Elisov, N.I. Ovchenkov, J. Scientific Bulletin of the MSTUCA 20-06, 36-43 (2017)

2. The national safety strategy of the Russian Federation until 2020. Decree of the President of the Russian Federation of 12 May 2009 № 537

3. Federal law № 16-FZ

4. R. Kemp, Ingenia magazine 16, 35-39 (2003) ISSN 1472-9768

5. E.A. Lavrukhina, Electronic Scientific Journal APRIORI, Series: The humanities 1, 110 (2016)

6. V.G. Popov, J. The World of Transport 3, 18-28 (2004)

7. D.V. Gontar, J. Novainfo 53-2 (2016) ISSN 2308-3689

8. Regulation on the organization of investigation and recording of traffic accidents and other events related to the violation of safety rules for the movement and operation of the railway transport on the infrastructure of JSC "RZD” (2017)

9. Provisions on the procedure for recording traffic accidents and other events related to the violation of safety rules for the movement and operation of the railway transport on the infrastructure of JSC "RZD” (2015)

10. V.G. Popov, On the analysis of the state and quantitative assessment of the safety level of train traffic on Russian railways, Proceedings of V SPC "Safety of train traffic" (MIIT, 2004)

11. Analysis of the state of traffic safety on the railways in Russia in 1998, Reports of the Department of traffic safety and ecology of the Ministry of Railways of the Russian Federation (Moscow, 1999-2006)

12. G.K. Zipf, Addison-Wesley 11, 574 (1949)

13. L.N. Kosarev, V.M. Rudanovsky, On the need for a new classification of traffic safety violations in railway transport, Proceedings of IV SPC "Safety of train traffic" (MIIT, 2003)

14. A.E. Kraskovsky, J. Railway Transport 5, 29 (2002)

15. Yu.L. Vorobyov, Strategic risks of Russia: assessment and forecast EMERCOM of Russia (Business express, Moscow, 2005) 